\title{
Identificación de interacciones selectoras en estudios etológicos y de psicología comparada
}

\section{Identification of selector interactions in etholofical and comparative psychology studies}

\author{
Gelacio Guzmán-Díaz ${ }^{a}$, Jesús Cisneros-Herrera ${ }^{b}$, Edgar Montes Castro $^{c}$
}

\begin{abstract}
:
Scientific analysis of objects and events from reality should be based on an analysis of shared characteristics of such objects and events. Particularly, in behavior experimental analysis, scientific study, among other elements, has its foundations on historic antecedents. In this paper, some studies related to ethological observation as well as comparative study of behavior as historic antecedents of the so-called selector function in the behavior functional taxonomy of Ribes and López (1985).
\end{abstract}

Keywords:

Interbahavioral Psychology, Behavior Functional Taxonomy, Selector Function, Compared Ethology and Psychology

\section{Resumen:}

El análisis científico de los objetos y eventos de la realidad debe partir de un análisis de características compartidas por tales objetos y eventos. En el caso particular del análisis experimental de la conducta, el estudio científico, entre otros elementos, está posibilitado por sus antecedentes históricos. En el presente escrito se analizan algunos estudios relacionados con la observación etológica y el estudio comparativo de la conducta como antecedentes históricos de la llamada función selectora de la taxonomía funcional del comportamiento propuesta por Ribes y López (1985) ${ }^{1}$.

\section{Palabras Clave:}

Psicología interconductual, Taxonomía funcional del comportamiento, Función selectora, Etología y Psicología comparada

El modelo de Ribes y López (1985) ${ }^{1}$ se enmarca en la tradición conductista, es decir, constituye una aproximación psicológica que reconoce al comportamiento de organismos e individuos como su objeto de estudio. El comportamiento psicológico se da dentro de una campo interconductual que implica la interacción entre la actividad de un organismo o individuo en relación con las funciones de los objetos, eventos $u$ otros organismos e individuos del ambiente, regulada por factores disposicionales tanto organísmicos como ambientales y posibilitada por diferentes medios de contacto (Kantor, 1959)2.
La propuesta taxonómica de Ribes y López (1985) ${ }^{1}$ reconoce cinco tipos cualitativos de interacción 0 funciones psicológicas: a) contextual; b) suplementaria; c) selectora; d) sustitutiva referencial; y e) sustitutiva no referencial. En la función selectora la actividad del organismo se vuelve autónoma con respecto a las propiedades fisicoquímicas de los eventos que varían momento a momento. En el nivel previo de organización de la conducta (función suplementaria), la relación entre dos eventos de estímulo (Ey-Ex) es condicional a la respuesta del organismo. En la interacción selectora hay un tercer evento, el estímulo selector (Es) que puede ser previo o simultaneo a la relación suplementaria. El evento

\footnotetext{
Autor de Correspondencia, Universidad Autónoma del Estado de Hidalgo, https://orcid.org/0000-0003-4869-8658, Email: gelacio_guzman@uaeh.edu.mx

b Universidad Autónoma del Estado de Hidalgo, https://orcid.org/0000-0003-1370-8086, Email: jesus_cisneros@uaeh.edu.mx ${ }^{c}$ https://orcid.org/0000-0001-7997-1149, Email: eduardomontes46@hotmail.com
} 
Es y la respuesta a dicho evento (Rs) determinan la funcionalidad de la relación suplementaria.

La psicología comparada del comportamiento y la etología surgieron a finales del siglo XIX y principios del siglo XX. Desde sus inicios ambas disciplinas se interesaron por cuestiones aparentemente distintas. Los etólogos se encargaron de la observación de los animales en su ambiente natural, en términos de la identificación de patrones conductuales propios de cada especie. Por otro lado, los psicólogos del comportamiento animal surgieron al mismo tiempo como una opción para el estudio de la interacción entre animales bajo situaciones restringidas, es decir, en laboratorios.

Con respecto a los estudios realizados por etólogos e investigadores de orientación zoológica, es menester señalar un inconveniente que complica severamente la interpretación del comportamiento animal en situaciones naturales en términos de las contingencias de ocurrencia y de función. Las contingencias de ocurrencia son las condiciones necesarias y suficientes para que ocurra físicamente un evento y las contingencias de función son las relaciones circunstanciales que se establecen entre las propiedades de los objetos de estímulo y las respuestas del organismo (Ribes, 1997) ${ }^{3}$. Específicamente, en la literatura destaca que las descripciones que hacen los etólogos sobre sus observaciones están, obviamente, determinadas por todo aquello que constituye un problema conceptualmente relevante en etología.

En un estudio realizado por Utami, Wich, Sterck, y Van Hooff $(1997)^{4}$, por ejemplo, se describen una serie de observaciones en torno de una población de orangutanes en su ambiente natural, en el contexto de la búsqueda de alimento y la competencia por conseguirlo. Dichos autores mencionaron varios elementos que potencialmente podrían participar como eventos intercambiables en la búsqueda de alimento: a) la abundancia de la comida, b) el número de orangutanes, c) el sexo de los conspecíficos, d) su edad y la de los demás orangutanes, e) el tipo de alimento, entre otras.

Específicamente, Utami y cols. $(1997)^{4}$ describieron la búsqueda de alimento en términos de la relación entre sólo dos variables (e.g. búsqueda de alimento y la abundancia de este), así como del efecto que podía tener una sobre la otra. Mencionan cómo los diferentes elementos pueden interactuar en conjunto sobre la búsqueda de alimento. Por ejemplo, afirman que si la comida es abundante los orangutanes no compiten por ella. Asimismo, señalan que la agresión entre los miembros de la manada es más común cuando se asientan en una zona que cuando están viajando. También indican que los orangutanes adultos son más eficientes en la búsqueda de alimento en comparación con los orangutanes jóvenes, así como que, en general, los orangutanes prefieren la fruta madura sobre la inmadura.
Estos elementos podrían formar parte de una interacción selectora, por ejemplo, la comida funciona como Ex, las conductas competitivas por la comida serian Ey's y la abundancia o no de la comida es el Es. Sin embargo, la realización de este análisis es difícil dada las descripciones de los autores la cual obedece a otros propósitos.

El mismo problema se puede identificar, por ejemplo, en el análisis etológico de la transmisión generacional del uso de herramientas en primates no humanos (Dindo, Stoinski \& Whiten, 2011 \& Tomasello, Davis-Dasilva \& Camak, 1987 $)^{5,6}$. El aprendizaje de uso de herramientas, mencionan estos autores, se establece por imitación y facilita la búsqueda y obtención del alimento en este tipo de especies. Sin embargo, aunque esta conducta involucra prácticas pre sociales entre animales para su transmisión, no se ofrecen detalles que permitan hacer un análisis en términos de la contingencia selectora e interpretar si en tales prácticas están involucrados procesos de tipo selector. Si quisiéramos hacer una interpretación en términos de la función selectora, tendríamos que saber bajo qué circunstancias los primates de sus estudios utilizan o no herramientas, si existe alguna correlación entre la comida y el tipo de herramientas a utilizar, si el aprendizaje sólo se da entre ciertos miembros de la especie o en todos ellos, de qué factores depende el uso de herramientas y su transmisión y cómo es que todos estos elementos se relacionan entre sí.

En el ámbito de la etología la ausencia de información pertinente hace difícil la identificación del tipo de interacciones funcionales selectoras, sin embargo, es posible traer a colación un ejemplo con respecto a la cacería que realizan las leonas. De acuerdo con Stander y Albon (1993) ${ }^{7}$, la comprensión de los hábitos sociales de los leones que tienen lugar durante la búsqueda de alimento, podría ser crucial para la interpretación de las causas evolutivas de la socialización. En los seres humanos las diferencias a partir de las culturas, religiones, patrones alimenticios, etc., dan como resultado una gran variedad de comportamientos que podrían compartirse entre miembros de una misma comunidad pero no entre comunidades. De acuerdo con dichos autores con los leones, así como con otras especies animales, se puede identificar algo semejante, ya que a pesar de que sean de la misma especie, los leones presentan comportamientos diferentes dependiendo del sistema ecológico en el que habitan. En esa medida, uno de los factores o variables más importantes que afectan el comportamiento de los leones es su ecosistema (Scheel \& Parker, 1991; Stander, 1991, Stander, 1992; Stander \& Albon, 1993) 8, 9, 10, 7.

En la presente revisión se hará referencia a dos hábitats distintos. Por un lado el Parque Nacional Serengueti (13.000 km²) -ubicado en Tanzania- y, por el otro, el 
Parque Nacional Etosha, situado en Namibia (22.270 $\mathrm{km}^{2}$ ). Cada uno de estos ecosistemas tiene características particulares que impactan sobre el comportamiento de los leones. En el caso particular de la búsqueda de alimento, entre tales características están: a) el área o terreno, b) la cantidad de leones y manadas de éstos, c) el clima, d) las distintas especies de presas, e) otras especies de predadores, f) la estación anual, g) la hora de cacería, h) la flora, entre otros. Al interior de la manada, factores adicionales que impactan sobre la cacería y la alimentación en grupo son: a) el número de miembros de la manada, b) la edad, c) el sexo, d) el parentesco, e) el peso de cada uno ellos, f) el tamaño, entre otros.

Los autores antes mencionados hicieron un análisis correlacional entre cada uno de estos factores y su impacto sobre la conducta individual de los leones que cazan en grupo. En la medida del tipo de variables, el reconocimiento de cada una en el contexto de las demás y del tipo de actividad de la que se trata, los estudios sobre los patrones alimenticios de los leones brinda un mayor número de elementos para reconocer interacciones de tipo selector en el comportamiento animal en situaciones naturales.

Una situación de caza cooperativa, de acuerdo con los autores, se da cuando dos o más leones comienzan a acechar a una presa con expresiones y posturas de alerta, para después seguirla y finalmente atraparla o intentar atraparla. En la mayoría de las ocasiones son las leonas la que realizan las cacerías, aunque, dependiendo de la presa, los leones también cazan algunas veces. No todas las cacerías que realizan las leonas parecen estar coordinadas. Sin embargo, de acuerdo con Stander $(1991)^{9}$, entre más grande es la presa mayor es el número de miembros de la manada que participan $y$, adicionalmente, es más probable que se adopten ciertas estrategias y roles que lleven a una cacería exitosa. Para Scheel y Parker $(1991)^{8}$, la participación en conjunto y coordinada de las leonas depende del tamaño de la presa, lo que lleva a suponer que, de alguna manera, inicialmente determinan si la cooperación es indispensable o no, en cuyo caso sólo observan. En términos de la función selectora podría decirse que el tamaño de la presa funciona como un Es que regula la relación entre la actividad de las leonas.

El análisis de Scheel y Parker $(1991)^{8}$, realizado en el Serengueti, poco nos dice acerca de cómo las leonas reconocen que tendrán o no éxito cazando en equipo. Sólo mencionan que el tamaño de la presa parece ser clave. Así pues, si la presa es pequeña no se necesitará más de una leona para una cacería exitosa. Si la presa es grande se necesitan más leonas, pero, si la presa es demasiado grande y la posibilidad de éxito es mínima, entonces no cooperarán. Adicionalmente, los autores mencionan que en la medida de que las manadas de leones recorren muchos kilómetros en busca de presas, es probable que en lugar de seguir estrategias de cooperación en realidad sólo participen las leonas disponibles en un momento determinado, o bien que participen sólo aquellas leonas con la suficiente energía para la cacería. Por otro lado, afirman que algunas leonas acompañan sólo algunas veces a otras durante la cacería, sin llevar a cabo ninguna actividad de ataque, "con el fin" de no ser detectadas como no participantes por las otras leonas. Para estos autores, las observaciones disponibles resultan insuficientes para reconocer si las leonas forman estrategias y roles cooperativos para la cacería.

En contraste, para Stander $(1992)^{10}$, quien realiza sus observaciones en Etosha, los grupos de cacería regularmente tienen la misma formación para cazar, siempre y cuando el tamaño de la presa así lo requiera. Este autor ha señalado que las leonas despliegan al menos tres tipos de estrategias de cacería. Los dos primeros corresponden a cazar en solitario o bien en grupo, pero de forma desorganizada. Sólo el tercer tipo correspondería a una caza cooperativa y coordinada, la cual definitivamente se da en función del tamaño de la presa. Esta cacería involucra un alto nivel de coordinación para la emboscada de la presa. Algunos leones (sin distinción de género) pueden abrir un abanico y encerrar a la presa, mientras otros esperan o avanzan lentamente. Todos los leones ven a la presa y a partir de ello ajustan sus propios movimientos, sin embargo, también ven a los demás miembros de la manada y sus movimientos, los que en igual medida determina lo que se debe hacer.

Tomando a la presa como punto de referencia, en la formación de abanico, algunas leonas parten de los costados; a estas leonas se les denomina "alas". Otras leonas inician el acecho desde el centro y por tal razón a estas últimas se les denomina "centros". Las leonas que ocupan el lugar de las alas tienen un rol de acecho y frecuentemente inician el ataque sobre la presa. Las leonas del centro, por otro lado, se desplazan distancias relativamente pequeñas y muy a menudo son quienes capturan a la presa. El autor menciona que no existen diferencias significativas entre la edad, el peso y el tamaño de las leonas con respecto a la posición que ocupan en la formación de ataque. Sin embargo, un análisis de sus datos muestra que, las leonas del centro son más longevas, pesadas y grandes. De hecho, dada cierta manada, generalmente cada leona ocupa repetidamente la misma posición en la formación de cacería, y aunque tal posición puede variar de acuerdo con la composición del grupo y la variación en la conducta de sus compañeras y de las presas, de acuerdo con Stander y Albon (1993) ${ }^{7}$, cuando las leonas cazan en su posición o rol favorito la oportunidad de éxito es mayor.

En otro reporte, Stander $(1991)^{9}$ observó a siete leonas que mostraban distintas preferencias por posiciones 
particulares de caza y se comportaban distintamente con las otras leonas. Estas siete leonas regularmente adoptaban el mismo rol dentro del grupo. Dos de las leonas observadas, la número 34 y la número 39 , regularmente cazaban juntas y tomaban las mismas posiciones, "ala derecha" y "centro", respectivamente. Además, en algunas ocasiones intercambiaban sus posiciones entre ellas. El propio autor señala que éste puede ser un ejemplo simple que sugiere que las leonas ajustan sus tácticas de cacería en respuesta a la presencia o ausencia y posición de los otros miembros del grupo de leonas cazadoras. Algunos de los datos que nos brinda Stander son el peso, la edad y el tamaño de las leonas. Como se mencionó anteriormente, las leonas del centro suelen ser más longevas y pesadas. La leona 34 que intercambiaba posición con la leona 39, era la leona más pesada en comparación con las otras leonas que también jugaban la posición de ala e incluso algunas del centro. Esto sugiere que el peso de la leona determina su rol de centro, debido a las funciones que tiene que realizar $y$, más importante, que el resto de las leonas son capaces de responder diferencialmente a esta situación y, en esa medida, adaptarse a las circunstancias. En términos de la función selectora el peso fungiría como Es, siendo una propiedad cambiante dependiendo de las leonas que conforman el grupo de caza.

De acuerdo con Stander $(1991)^{9}$, existe evidencia suficiente para decir que las leonas tienden a cooperar para lograr una caza exitosa, así como que la cooperación y el éxito depende de varios de los factores ya mencionados, es decir el tamaño y tipo de presa, el tamaño del grupo, el terreno, etc. Al no tener un episodio concreto es difícil afirmar que en la caza cooperativa de las leonas estén implicadas interacciones de tipo selector, sin embargo, a partir de las observaciones etológicas existentes se pueden inferir ciertas configuraciones.

En la terminología de la propuesta taxonómica de Ribes y López (1985) ${ }^{1}$, la presa, que constituye el eje de referencia inicial, correspondería al llamado estímulo contextualizador o Ex. Para contactarlo, es decir para cazarlo exitosamente, cada una de las leonas tendría que asumir alguna de las diferentes posiciones de ataque en función de la posición de la presa, pero igualmente de la posición de las demás leonas y sus características. En un caso hipotético, la leona A observa una presa a la distancia. Con sus gestos y postura alerta a sus compañeras, lo que sirve como estímulo selector para las otras leonas, quienes no han contactado visualmente a la presa en cuestión. La leona B identifica las posturas y gestos de la leona A, lo que le indica que hay una presa y, por tanto, dirige su vista hacia donde se encuentra la presa y despliega algunas conductas preparatorias para el asecho (probablemente algunas veces incluso antes de ver a la presa). La leona B identifica a la presa y de acuerdo con las características de ésta, es decir si es grande y pesada, pequeña y ligera e incluso probablemente si es grande pero ligera ( $\mathrm{y}$ en esa medida veloz) o pequeña y pesada (y en esa medida lenta), participará o no en la cacería. Lo que trata de resaltarse para este momento es que la postura y gestos de la leona A son una señal de la presa, pero por sí sola no señala que habrá cacería grupal. Se necesita de otros segmentos de estímulo para darle funcionalidad a dicha señal.

Tales segmentos de estímulo, que podrían conceptualizarse como estímulos de segundo orden (véase caso 4 de la función selectora), son, por un lado, el tipo de presa y sus características (cebra, avestruz, ñu, antílope, pequeño, mediano, grande, muy grande, cría, joven, adulto, longevo, ligero, pesado, muy pesado, etc.) $y$, por el otro, el resto de las leonas disponibles (digamos leona $\mathrm{X}$, leona $\mathrm{Y}$ y leona $Z$ ) y sus características. Este Es2 también varía, pues la leona B podría o no participar dependiendo del comportamiento variante de sus compañeras. Por ejemplo, si la leona $X$ por saciedad $u$ otra razón no participara en la cacería, la leona $B$ optaría por hacerlo. Por otra parte si la leona $X$ sí participa en la cacería y es más grande, pesada y longeva ocupará la posición centro por lo que la leona B ocuparía la posición de ala. Si estas características no se cumplieran en la leona $\mathrm{X}$, las posiciones podrían o no intercambiarse.

Como es evidente, el análisis de episodios de tipo selector a partir de las observaciones etológicas en ambientes naturales requiere un alto grado de interpretación. Al menos en el caso de las leonas, no podemos concluir que la cacería cooperativa implique o no configuraciones comportamentales de tipo selector. $Y$ aunque la metodología puramente observacional seguida en este tipo de estudios no es el único factor que impide llegar a dichas conclusiones, la investigación de laboratorio bajo circunstancias que simulan interacciones de supervivencia y/o socialización en animales podría ayudarnos a descartar este factor metodológico implicado en la etología. Las aproximaciones realizadas bajo laboratorio permiten manipular las variables que interactúan en la situación y evitar variables extrañas que pudiesen oscurecer en el análisis.

Daniel (1942) ${ }^{11}$ indagó experimentalmente acerca del desarrollo de la conducta cooperativa en animales. Específicamente, dos ratas tenían que "ayudarse mutuamente" para evitar un choque eléctrico y poder consumir alimento. En el estudio se conformaron seis parejas de ratas. La situación experimental consistió en una caja experimental con piso de rejilla electrificado. En uno de los extremos de la caja se colocó una plataforma que al ser presionada por todo el peso de la rata, cancelaba la electricidad del piso de rejilla. En el otro extremo de la caja se encontraba el alimento. Dos ratas eran introducidas al mismo tiempo en la cámara 
experimental. La "ayuda mutua" consistía en que mientras una de las ratas permanecía en la plataforma para cancelar la descarga eléctrica, la otra rata podía comer. Posteriormente ambas ratas debían intercambiar sus roles y el éxito de la cooperación dependía de que ambas participaran de manera coordinada.

Antes de que las ratas fueran introducidas a la situación experimental cooperativa, recibieron un entrenamiento individual de tres fases. En la primera fase se colocó a cada rata en la caja experimental con el piso sin electrificar y con acceso libre al alimento. Esta fase consistió en dos ensayos diarios de 450-s cada uno, durante ocho días. Después del octavo día en las condiciones anteriores, comenzó la segunda fase preliminar, en la que cada rata fue introducida a la caja con el piso de rejilla electrificado y permanecía ahí hasta que pisara la plataforma y se mantuviera en ella por 30s. Esta fase tuvo 20 ensayos en un solo día. Por último, la tercera fase previa a la fase cooperación, consistió en una mezcla de 20 ensayos con y sin piso electrificado durante cinco días. En los ensayos con piso electrificado la rata debía aprender a ir hacia la plataforma y permanecer ahí 30s. En los ensayos con piso no electrificado las ratas aprendían a alimentarse sin recibir descargas eléctricas.

Después del entrenamiento preliminar se expuso a las ratas a la situación de cooperación. Durante esta fase se colocó a las dos ratas dentro de la caja experimental, provista con un dispensador de comida ubicado al extremo opuesto de la plataforma que interrumpía la descarga del piso electrificado. Así, al menos una de las ratas debía estar sobre la plataforma para evitar la descarga eléctrica y la otra rata pudiese ir hasta el alimento y consumirlo. En general, en el estudio se observó que cuatro de las seis parejas de ratas mostraron una ejecución alternada, es decir, desplegaron actividades coordinadas que les permitieron evitar los choques eléctricos y comer.

En la identificación de los componentes de las relaciones de contingencia de la función selectora, por un lado, tenemos una interacción suplementaria en lo que a la evitación del choque eléctrico se refiere y, por el otro, una interacción puramente contextual en la que las ratas simplemente se orientan y desplazan hacia el alimento. La respuesta suplementaria (Ry) consiste en subirse a la plataforma para interrumpir la descarga eléctrica. El alimento está presente durante todo el experimento, por lo que ninguna respuesta de las ratas produce, acelera o retarda la presentación de aquel. Aunque la presión de la plataforma hace posible que se pueda tomar el alimento sin recibir una descarga, esta respuesta no es suplementaria con respecto a la presentación de la comida.

La respuesta contextual de la rata $A$ radica en aproximarse a la comida mientras la otra rata, la $\mathrm{B}$, está sobre la plataforma, es decir, la rata $B$ funciona como estímulo que señala a la rata $A$ que se aproxime al comedero. Asimismo, la posición de la rata $\mathrm{B}$, estar o no sobre la plataforma, constituye el estímulo discriminativo de la respuesta suplementaria para la rata $A$, es decir, si la rata $B$ no está encima de la plataforma la rata $A$ debe presionarla para evitar el choque eléctrico. El mismo análisis aplicaría si la rata $B$ fuese el eje de referencia. Así, el comportamiento de ninguna de las dos ratas puede considerarse como un caso de función selectora ya que la respuesta suplementaria, presionar la plataforma para escapar del choque, sólo está señalada por la presencia o ausencia de la otra rata sobre la plataforma, es decir, la otra rata funciona como estímulo discriminativo pero su funcionalidad no cambia. Así pues, en el mejor de los casos, en este tipo de situación experimental sobre conducta cooperativa en ratas se presentan interacciones suplementarias y contextuales independientes, más que un verdadero episodio selector.

Otra línea de investigación que tiene que ver con la comunicación y el intercambio para o pre social en animales son los estudios experimentales sobre conducta cooperativa y la reciprocidad en ratas. Rutte y Taborsky (2008) ${ }^{12}$ y más recientemente Schneeberger, Dietz, y Taborsky $(2012)^{13}$ estudiaron la reciprocidad utilizando ratas como sujetos experimentales. Para los autores, el tipo de interacciones que tienen lugar bajo sus paradigmas experimentales para analizar la reciprocidad son muestra de la evolución de la cooperación. De acuerdo con dichos autores, la reciprocidad altruista o cooperación recíproca, consiste en dar y recibir ayuda entre individuos que no necesariamente están relacionados socialmente, es decir, que no han tenido interacciones sociales previas. La "decisión" de cooperar o no hacerlo está basada en la expectativa de ser ayudado en un futuro. La reciprocidad puede ser directa o indirecta; la primera se refiere a cuando $A$ recibe ayuda de $B$ porque $B$ fue ayudado antes por $A$. En la reciprocidad indirecta, $A$ recibe ayuda de $B$ porque $B$ fue ayudado por $C$ anteriormente.

Tanto Rutte y Taborsky $(2007)^{12}$ como Schneeberger, Dietz, y Taborsky $(2012)^{13}$ utilizan la misma situación experimental para el estudio de la conducta cooperativa en ratas. Este escenario consiste en una caja experimental dividida en dos secciones -A y B-por un panel transparente. Cada espacio es ocupado por una rata, Rata A y Rata B. Dentro del compartimiento en el que es colocada la Rata A hay un pequeño bastón, que al ser jalado por la rata introduce en cada compartimiento un recipiente en donde puede o no haber alimento. La rata que al jalar el bastón introduce los recipientes es denominada rata "focal". En la situación altruista se deposita alimento sólo en el recipiente contrario a aquel en el que está ubicado del bastón. De esta forma, si el 
bastón se encuentra en la sección A y es jalado por la rata focal, solo la rata del segundo compartimiento, es decir la rata $\mathrm{B}$, recibirá alimento. La rata que recibe el alimento por el comportamiento de la rata focal es denominada rata "compañera". En un segundo momento, se intercambian el bastón y el alimento entre los compartimentos, de modo que ahora es la rata $B$ la que debe jalar el bastón para proporcionarle alimento a la rata A. Antropomorfizando, diríamos que las ratas intercambian sus roles y que de esta forma las ratas se comportan recíprocamente.

Rutte y Taborsky $(2007)^{12}$ estudiaron si la conducta cooperativa de las ratas podría estar influenciada por la experiencia de haber recibido ayuda o no por otra rata. Antes de exponer a las ratas a la situación de cooperación o no cooperación, hubo dos pre-entrenamientos. El primero consistió en el entrenamiento individual de la respuesta de jalar el bastón para obtener la comida que había en el recipiente. En el segundo pre-entrenamiento, que estuvo vigente durante 70 sesiones, cada una de las ratas fue entrenada a jalar el bastón para acercarle el alimento a su compañera en el otro compartimento. Después de 35 sesiones el bastón se cambió de compartimento, de modo que la rata originalmente focal ahora sería rata compañera.

Ya en la fase experimental de altruismo, todas las ratas pre entrenadas fueron utilizadas como ratas focales $y$ fueron expuestas durante seis sesiones a una condición de reciprocidad con seis ratas distintas; tres ratas que tenían el mismo pre entrenamiento y otras tres ratas que eran experimentalmente ingenuas. Para estas últimas, el bastón estaba asegurado de tal suerte que jalarlo no acercara el contenedor de alimento a la rata focal. El orden de presentación de las seis ratas que fungieron como compañeras fue aleatorizado para cada rata focal, es decir, para cada rata focal las situaciones de cooperación o no cooperación fueron programadas al azar.

Los resultados mostraron que las ratas focales cooperaban más a menudo si su experiencia inmediatamente anterior había sido con una rata compañera que posteriormente fungió como focal, que si su experiencia inmediatamente anterior había sido con una rata compañera experimentalmente ingenua. De acuerdo con los autores, estos resultados indican que la cooperación recíproca no necesariamente se basa en un conocimiento específico del compañero, sino en una experiencia de cooperación.

Schneeberger, Dietz, y Taborsky $(2012)^{13}$ realizaron dos experimentos adicionales en el mismo tenor. En el primero manipularon el esfuerzo de respuesta por ayudar, variando la resistencia del bastón que tenía que ser jalado para introducir la comida. Cada nivel de resistencia del bastón se correlacionó con una señal visual, bajo el supuesto de que ello les permitiría "decidir" si ayudaban o no dependiendo del costo que les implicaría jalar el bastón. En el segundo experimento manipularon los "niveles de necesidad social" de las ratas que fungían como compañeras, lo que simplemente se refería a privar de alimento o no a tales ratas. Se esperaba determinar si las ratas focales ayudaban de forma diferencial a su compañera dependiendo de si ésta estaba o no hambrienta, lo que se suponía podía ser inferido por la rata focal a partir de la actividad general de la rata compañera. En el primer experimento las ratas fueron previamente entrenadas de forma individual a jalar el bastón para recibir comida. Las ratas que no aprendían esta respuesta fueron eliminadas del experimento. En una segunda fase se entrenó a las ratas aumentando la resistencia del bastón de 1 a 5 néwtones. Como se señaló anteriormente, cada valor de resistencia del bastón se correlacionó con un estímulo visual particular. A partir de estos preentrenamientos, las ratas fueron familiarizadas con la situación de reciprocidad. Dos ratas fueron colocadas en la caja y sólo una tenía acceso al bastón. La rata compañera recibía alimento por las respuestas de la rata focal, y en una sesión posterior los papeles eran intercambiados.

Posteriormente, todas las ratas entrenadas fueron expuestas a dos diferentes situaciones sociales en secuencia aleatoria: En la primera había una situación cooperativa en la que cada rata era focal y posteriormente compañera en dos bloques de siete minutos, respectivamente. En la segunda fase no había posibilidad de comportamiento reciproco, es decir, una rata era focal y la otra era compañera y estos roles no se intercambiaban.

Después de estas dos fases, de cooperación y de no cooperación, el bastón estuvo disponible para la rata que había realizado el papel de focal en la última fase, teniendo ahora la posibilidad de jalar el bastón para alimentar a la rata compañera, que en la fase anterior había jugado ese mismo rol. Los roles se intercambiaron después de siete minutos y al interior de los mismos los diferentes valores de resistencia del bastón y sus correspondientes señales se presentaron de forma aleatoria. La frecuencia con la que jalaron el bastón y la latencia para comenzar a jalarlo en cada valor de resistencia fueron usados como medidas de la propensión de las ratas focales para ayudar a su compañera.

En el segundo experimento las ratas que jugaron el rol de compañeras fueron privadas de comida durante la noche anterior a la situación experimental, de modo que estuvieran hambrientas para el experimento. Ambas ratas, la focal y la compañera, se expusieron a la misma situación que en el experimento anterior, es decir, sus roles se intercambiaron después de siete minutos, pero la resistencia del bastón se mantuvo en 1 newton. Adicionalmente, antes de que las ratas focales fueran expuestas a la situación de reciprocidad con la rata 
privada de alimento, enfrentaron la misma situación con una tercera rata no privada de alimento, con la finalidad de comparar si había diferencias entre ayudar a una rata con o sin necesidad de alimento.

En el primer experimento los autores encontraron que las ratas jalaron más veces el bastón para una rata cooperadora que para una rata no cooperadora. El número de veces que jalaron el bastón disminuyó con el incremento en el costo de respuesta, mientras que la latencia de la respuesta de jalar el bastón incrementó cuando se trataba de ayudar a una rata no cooperadora. En el segundo experimento, en el que las ratas compañeras estaban o no privadas de comida, se observó que las ratas focales jalaron más veces el bastón cuando las ratas compañeras estaban privadas de alimento que cuando no estaban privadas.

Aunque los autores no reportaron si la conducta de una rata saciada es diferente de la de una rata privada de comida, argumentaron que tal diferencia podría ser la razón por la que las ratas focales reconocen el estado de su compañera. De ser este el caso, el comportamiento de las ratas focales, siempre y cuando se atienda al comportamiento de las ratas compañeras y otras de las variables de los estudios, podría ejemplificar comportamiento de tipo selector ya que implica la variación ecológica de eventos organísmicos con propiedad de estímulo, tal como Ribes y López $(1985)^{1}$ sugirieron para esta función psicológica. La respuesta Ryx de jalar el bastón, tendría o no lugar dependiendo de, por un lado, los estímulos ambientales, por ejemplo las señales visuales correlacionadas con cada valor de resistencia (Ey), y, por el otro, los estados organísmicos y actividades correlacionadas de la rata compañera, así como si ésta ha sido o no recíproca anteriormente en la producción de alimento (Es's).

En conclusión, al menos en el caso de la investigación etológica, la revisión anterior cuestiona la necesidad de manejar los estudios de tal área de investigación como antecedente históricos de la función selectora, en la medida de que la posibilidad de identificar episodios de dicha complejidad funcional está severamente limitada. La investigación experimental en psicología comparada, parece confirmar algunas de las características señaladas por Ribes y López (1985) ${ }^{1}$ en torno de tal función psicológica. La dificultad para identificar tal o cual tipo de episodios interactivos desde una perspectiva psicológica a partir de las observaciones etológicas, probablemente pueda superarse mediante una aproximación multidisciplinaria. Es decir al abordaje conjunto de un fenómeno entre dos disciplinas, en donde el prefijo indica la dominancia teórica del abordaje, mientras que el complemento indica regularmente una aportación metodológica a su estudio (Ribes, 2010) ${ }^{14}$.

\section{Referencias}

[1] Ribes, E. y López, F. (1985). Teoría de la conducta. México: Trillas.

[2] Kantor, J. R. (1959). Interbehavioral psychology: A sample of system construction. Granville: Principia Press.

[3] Ribes, E. (1997). Causality and contingency: Some conceptual considerations. The Psychological Record, 47, 619-639.

[4] Utami, S., Wich, A., Sterck, E. \& Van Hooff, J. (1997) Food competition between wild orangutans in large fig trees. International Journal of Primatology, 18, 909-927.

[5] Dindo M, Stoinski T, \& Whiten A. (2011). Observational learning in orangutan cultural transmission chains. Biol Lett, 7, 181-183.

[6] Tomasello, M., Davis-Dasilva, M., \& Camak, L. (1987). Observational learning of tool-use by young chimpanzees. Human Evolution, 2, 175183.

[7] Stander, P. \& Albon, S. (1993). Hunting success of lions in a semi-arid environment. Symposia of the Zoological Society, 65, 127-143.

[8] Scheel, D. \& Packer, C. (1991). Group hunting behaviour of lions: a search for cooperation. Animal Behaviour, 41, 697-709.

[9] Stander, P. (1991). Demography of lions in the Etosha National Park. Madoqua 18, 1-9.

[10] Stander, P. (1992). Cooperative hunting in lions: the role of the individual. Behavioral Ecology and Sociobiology, 29, 445-454.

[11] Daniel, W. (1942). Cooperative problem solving in rats. The Journal of Comparative Psychology, 34, 361-368.

[12] Rutte, C. \& Taborsky, M. (2008). The influence of social experience on cooperative behavior. Behavioral Ecology and Sociobiology, 62, 499-505.

[13] Schneeberger, K., Dietz, M., \& Taborsky, M. (2012). Reciprocal cooperation between unrelated rats depends on cost to donor and benefit to recipient. Evolutionary Biology, 12, 1-7.

[14] Ribes, E. (2010). Lenguaje ordinario y lenguaje técnico: un proyecto de currículo universitario para la psicología. Revista Mexicana de Psicología, 27, 55-64. 